flgenda

\section{AGENDA NATIONAL}

\section{NOVEMBRE}

Journées Internationales de Biologie (/IB)

Du 3 au 6 novembre 2004

à Paris-La Défense.

Pour le programme et l'inscription, voir la page consacrée à

l'événement sur le site du syndicat des biologistes (sdbio.fr) ou l'agenda de BioTribune.com.

$47^{\text {éme Journée de l'Hôpital }}$

Claude Bernard

Le 19 novembre 2004 à Paris. Le thème retenu est "Résistance bactérienne aux antibiotiques : déterminants et maîtrise". Renseignements : www.b-c-a.fr

\section{DECEMBRE}

$38^{\text {emes }}$ Journées de Biologie Praticienne

Le 10 et II décembre 2004 à

Paris.

Renseignements :

www.feuilletsdebiologie.com

gème Journée annuelle du GEMHEP : les hépatites $C$ et $B$ Le 16 décembre 2004 à Paris. Renseignements : www.b-c-a.fr

\section{IANVIER}

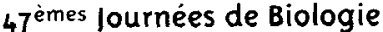

Clinique Necker

Du 17 au 19 janvier 2005 à

Paris.

Renseignements et inscription :

Agence DV

Tél. : ol 45665342

Mail : dvl@wanadoo.fr

\section{MARS}

\section{MEDEC 2005}

Du 15 au 18 mars 2005 à Paris. Le salon pour les professionnels de la médecine.

Renseignements :

www.lemedec.com

\section{ALFEDIAM}

Du 22 au 26 mars 2005 à Lyon. Congrès annuel de l'ALFEDIAM (Association de langue française pour l'étude du diabète et des maladies métaboliques). Programme et inscription: www.alfediam.org

\section{AVRIL}

Journées Francophones de pathologie digestive Du 2 au 6 avril 2005 à Paris. Organisées par la SNFGE (Société Nationale Française de Gastro-Entérologie).

Renseignements : www.snfge.org

$7^{\text {èmes }}$ Journées

Francophones de Virologie Du 28 au 29 avril 2005 à Paris. Renseignements : www.b-c-a.fr

\section{AGENDA INTERNATIONAL}

\section{NOVEMBRE}

\section{MEDICA 2004}

Allemagne

Du 24 au 27 novembre 2004 à Dusseldorf.

La foire internationale de l'équipement médical.

Renseignements :

www14.medica.de

\section{DECEMBRE}

RICAl 2004 couplée à

l'European Congress of

Chemotherapy and

Infections (ECC)

France

Du jer $^{\text {au }} 3$ décembre 2004 à

Paris.

Renseignements:

www.ricai.org

\section{ASH Annual Meeting}

Etats-Unis

Du 4 au 7 décembre 2004 à San Diego (Californie). 46 ème congrès annuel de l'American Society of Hematology.

Renseignements

www.hematology.org/meeting

\section{FEVRIER}

$12^{\text {th }}$ Conference on

Retroviruses and

Opportunistic Infections

Etats-Unis

Du 8 au II février 2005 à San

Fransisco (Californie).

Renseignements

www.retroconference.org

AVRIL

$15^{\text {th }}$ European Congress of

Clinical Microbiology and Infectious Diseases

Danemark

Du 2 au 5 avril 2005 à

Copenhague.

Renseignements :

www.akm.ch

\section{FORMATION CONTINUE}

NOVEMBRE

Reproduction (BP.G).

Exploration biologique de la fonction de reproduction Le 18 et 19 novembre 2004

à Paris.

Renseignements et inscription : www.bioformation.org

Oncologie (BP.H).

Marqueurs tumoraux cellulaires et sériques

Le 25 et 26 novembre 2004

à Paris.

Renseignements et inscription : www.bioformation.org

\section{DECEMBRE}

L'immunohématologie en pratique quotidienne Le 2 décembre 2004 à Reims. Renseignements et inscription : www.biologie-prospective.org

Virologie (BP.I). Hépatites virales et Sida - Rôle du laboratoire dans le diagnostic des infections virales

Le 2 et 3 décembre 2004 à Paris.

Renseignements et inscription : www.bioformation.org

Exploration biologique du diabète. Données 2004 Le 6 décembre 2004 à Paris Renseignements et inscription : www.corata.org

Tél. : ol 44412375

Fax : 0144412388

acorataacochin.univ-paris5. fr

Détermination du risque de trisomie 21 foetale Apport des marqueurs sériques maternels Le 9 et 10 décembre 2004 à Paris.

Renseignements et inscription : www.bioformation.org

Dyslipidémies et coronaires Le 14 décembre 2004 à Paris. Renseignements et inscription : www.corata.org

Tél. : ol 44412375

Fax : ol 44412388

Mail : acorata®cochin.univ-pariss.fr 